\title{
Superposition of small shear deformations on large uniaxial extensions for viscoelastic materials*
}

\author{
L. J. Zapas \\ National Measurement Laboratory, National Bureau of Standards, Washington, D.C. \\ 20234, USA \\ and Alan Wineman \\ Department of Mechanical Engineering and Applied Mechanics, The University of \\ Michigan, Ann Arbor, Michigan 48109, USA \\ (Received 23 August 1984)
}

\begin{abstract}
The equation for small amplitude torsional response superposed on a finite axial stretch history is derived for a material modelled by the BKZ constitutive equation. For the case of a uniaxial step stretch history, this equation contains a term generalizing the well known Rivlin result for nonlinear elasticity plus two additional terms. The equation is used to analyse a recent experiment which studies the relation between the period of free torsional oscillations, axial stretch and axial force in a material undergoing stress relaxation. It is shown that a $10 \%$ discrepancy which arises when the data is related by Rivlin's formula can be accounted for by the two additional terms associated with the BKZ formulation. Finally, an expression for the complex modulus for forced torsional oscillations is derived.
\end{abstract}

(Keywords: shear deformations; viscoelastic materials; torsional response; stretch history)

\section{INTRODUCTION}

During the past few years, an interest has risen in the torsional dynamic response of polymeric rods which have been subjected to large uniaxial stretching ${ }^{1-3}$. For elastic materials, Rivlin ${ }^{4}$ showed that the torsional stiffness under infinitesimal angular deformations is independent of the strain energy density function and can be expressed entirely in a known relation between the axial force and the axial stretch ratio. This relation was verified experimentally by Rivlin and Saunders ${ }^{5}$ for vulcanized rubber. Analogous results for a general nonlinear viscoelastic material have been only partially obtained. In ref. 2 , the equation for small deformations superposed on an axial step stretch was derived for general materials. Some observations were made on the case of superposed shear strains. It was shown that the generalizations of Rivlin's formula for torsional rigidity would still hold when a superposed step shear strain was applied at the same time as the axial step stretch. However, the extension of Rivlin's formula to other shearing histories was not discussed.

In the present work, we study the small amplitude torsional dynamic response in the presence of large axial stretching when viscoelasticity is described by the BKZ fluid model ${ }^{6}$. The results presented in the following section show that two extra terms are needed for the proper description of the torsional response beyond the term for the elastic case. These depend on the axial stretch ratio and the shear modulus for linear viscoelastic response from the undeformed state. Consequently, it is not

* Presented at the 49th Annual Meeting of the Society of Rheology, 22-26 October 1978 possible to establish a relation analogous to Rivlin's formula, i.e. which does not depend on material properties, except in the case mentioned above.

In ref. 1, it was conjectured that if Rivlin's formula were valid in the presence of axial stress relaxation, then the period of free torsional oscillations in a prestretched bar should satisfy a specific relation in terms of the axial stretch and the axial force. It is shown later that a $10 \%$ discrepancy between the data presented in ref. 1 and this relation can be accounted for by the extra terms derived in the following section.

Forced torsional oscillations are treated in the forced vibration' section. An expression for the complex shear modulus is derived which shows the dependence on the axial stretch ratio and on time due to axial stress relaxation.

\section{TWISTING MOMENT-TWIST ANGLE RELATION}

Since the effects to be considered are in the non-linear range of material response, the $\mathrm{BKZ}$ model has been selected for this analysis. It has been successful in the modelling of viscoelastic behaviour for a variety of deformations in the nonlinear region.

The length, inner and outer radii of the undeformed cylindrical specimen are $L_{0}, a_{0}$ and $b_{0}$, respectively. At time $s>0$, its inner and outer surfaces are still cylindrical, its length is $L(s)$ and the relative rotation between the ends is $\phi(s)$. The point-wise description of the deformation defines a controllable motion for which it is well known that the equations of quasistatic motion can be satisfied 
for any constitutive equation. The motion is also such that the cylinder can be regarded as an assemblage of material elements each of which undergoes uniaxial extension and simple shear. This enables us to first discuss the response of a typical material element, and then to consider the complete cylinder.

The element in its deformed and undeformed states and a set of Cartesian coordinate axes are shown in Figure 1. The $x_{1}, x_{2}$ and $x_{3}$ axes of the element are associated with the axial, radial and tangential directions in the cylinder. Let $x_{\mathrm{i}}(s)$ denote the coordinates of a point at time $s$, $-\infty s<t$. The element is undistorted for $s<0$. For $s \geqslant 0$, the element is regarded as having undergone uniaxial extension along the $x_{1}$ axis followed by simple shearing in the $x_{3}$ direction. The resultant motion is described by the expressions:

$$
\begin{gathered}
x_{1}(s)=\lambda(s) x_{1}(0), \quad x_{2}(s)=\lambda(s)^{-1 / 2} x_{2}(0), \\
\mathrm{x}_{3}(\mathrm{~s})=\lambda(\mathrm{s})^{-1 / 2} \mathrm{x}_{3}(0)+K(s) \lambda(s) x_{1}(0)
\end{gathered}
$$

The shear $K(s)$ of a material element at radius $r$ in the deformed state, denoted by $K(r, s)$, is expressed in terms of the length and relative rotation between the ends by:

$$
K(r, s)=r \frac{\phi(s)}{L(s)}=\frac{r \phi(s)}{L_{0} \lambda(s)}
$$

The procedure for computing the stresses associated with this deformation using the $\mathrm{BKZ}$ constitutive equations was outlined by Bernstein 7 . This requires that the position of a point at time $s$ be expressed in terms of its position at time $t$. For the present case, the appropriate relation is given by:

$$
\left.\begin{array}{c}
x_{1}(s)=\frac{\lambda(s)}{\lambda(t)} x_{1}(t) \quad x_{2}(s)=\sqrt{\frac{\lambda(t)}{\lambda(s)}} x_{2}(t) \\
x_{3}(s)=\sqrt{\frac{\lambda(t)}{\lambda(s)}} x_{3}(t)+x_{1}(t) K(s) \frac{\lambda(s)}{\lambda(t)}-K(t) \sqrt{\frac{\lambda(t)}{\lambda(s)}}
\end{array}\right\}
$$

The deformation gradients for motion (3) and its inverse are:

$$
\underset{\sim}{\theta}(t, s)=\left[\partial x_{i}(t) / \partial x_{j}(s)\right]=\left[\begin{array}{ccc}
\bar{\lambda} & 0 & 0 \\
0 & \bar{\lambda}^{-1 / 2} & 0 \\
\bar{K} & 0 & \bar{\lambda}^{-1 / 2}
\end{array}\right]
$$

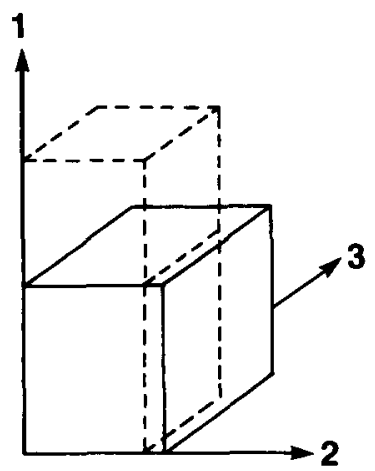

(a)

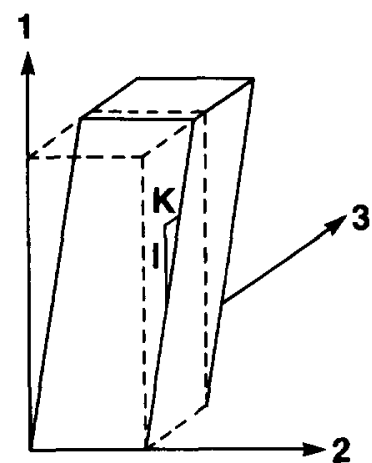

(b)
Figure 1 Assumed deformation of a material element, (a) extension along $x_{1}$ axis from undeformed state; (b) shearing in $x_{3}$ direction from extended state

$$
\underline{\theta}^{-1}(s, t)=\left[\partial x_{i}(s) / \partial x_{j}(t)\right]=\left[\begin{array}{ccc}
\bar{\lambda}^{-1} & 0 & 0 \\
0 & \bar{\lambda}^{1 / 2} & 0 \\
\bar{K} \bar{\lambda}^{-1 / 2} & 0 & \bar{\lambda}^{1 / 2}
\end{array}\right]
$$

where

$$
\bar{\lambda}=\frac{\lambda(t)}{\lambda(s)} \quad \bar{K}=K(t) \frac{\lambda(t)}{\lambda(s)}-K(s) \sqrt{\frac{\lambda(s)}{\lambda(t)}}
$$

The BKZ constitutive equation is expressed in terms of the left relative Cauchy-Green tensor and its inverse, which are:

$$
\begin{gathered}
\underset{\sim}{B}(t, s)=\underset{\sim}{\theta}(t, s) \underset{\sim}{\theta^{\mathrm{T}}}(t, s)=\left[\begin{array}{ccc}
\bar{\lambda}^{2} & 0 & \bar{\lambda} \bar{K} \\
0 & \bar{\lambda}^{-1} & 0 \\
\bar{\lambda} \bar{K} & 0 & \bar{K}^{2}+\bar{\lambda}^{-1}
\end{array}\right] \\
B^{-1}(t, s)={\underset{\sim}{\theta}}^{-\mathrm{T}}(t, s) \theta_{\sim}^{-1}(t, s)=\left[\begin{array}{cccc}
\bar{\lambda}^{-2}+\bar{K}^{2} \bar{\lambda}^{-1} & 0 & -\bar{K} \\
0 & \bar{\lambda} & 0 \\
-\bar{K} & 0 & \bar{\lambda}
\end{array}\right]
\end{gathered}
$$

The corresponding invariants are:

$$
\begin{aligned}
& I_{1}(t, s)=\operatorname{tr} \underset{\sim}{B}=\bar{\lambda}^{2}+2 \bar{\lambda}^{-1}+\bar{K}^{2} \\
& I_{2}(t, s)=\operatorname{tr}{\underset{\sim}{B}}^{-1}=2 \bar{\lambda}+\bar{\lambda}^{-2}+\bar{K}^{2} \bar{\lambda}^{-1}
\end{aligned}
$$

The $\mathrm{BKZ}$ constitutive equation is:

$$
\sigma=-p l+\int_{-\infty}^{t}\left\{U_{1} \underset{\sim}{B}(t, s)-U_{2} \underset{\sim}{B^{-1}}(t, s)\right\} \mathrm{d} s
$$

where $p$ is the undetermined reaction to incompressibility. $U_{1}$ and $U_{2}$ are a set of material dependent functions defined in terms of a scalar potential function $U$ by:

$$
U_{x}=\frac{\partial U}{\partial I_{x}}\left(I_{1}(t, s), I_{2}(t, s), t-s\right), \quad \alpha=1,2
$$

The cylinder is at rest for $-\infty<s<0$. A uniaxial stretch hsitory is applied at $s=0$. The description of the experiment in ref 1 implies that there is a time interval $t_{1}$ until the torsional deformation is applied. Thus, for each time $t>t_{1}$, the stretch and shear histories are specified on three distinct time intervals by the expressions:

$$
\begin{gathered}
\lambda(s)=1, \quad K(s)=0, \quad-\infty<s<0 \\
\lambda(s)>1, \quad K(s)=0, \quad 0 \leqslant s \leqslant t_{1} \\
\lambda(s)>1, \quad K(s) \text { arbitrary, } \quad t_{1} \leqslant s \leqslant t
\end{gathered}
$$

By equations (5), (7) and (10), the invariants defined for $s$ on the first interval, $-\infty<s<0$, depend only on $\lambda(t)$ and $\mathrm{K}(\mathrm{t})$. Their reduced expressions, denoted by $\hat{I}_{x}(t)$ are given by:

$$
\begin{aligned}
& I_{1}(t, s)=\hat{I}_{1}(t)=\lambda^{2}(t)+2 \lambda^{-1}(t)+K^{2}(t) \lambda^{2}(t) \\
& I_{2}(t, s)=\hat{I}_{2}(t)=2 \lambda(t)+\lambda^{-2}(t)+\lambda(t) K^{2}(t)
\end{aligned}
$$

For the intervals $0 \leqslant s \leqslant t_{1}$ and $t_{1} \leqslant s \leqslant t$, the invariants can be written as:

$$
I_{1}(t, s)=\left(\frac{\lambda(t)}{\lambda(s)}\right)^{2}+2\left(\frac{\lambda(s)}{\lambda(t)}\right)+\bar{K}^{2}
$$


$I_{2}(t, s)=2\left(\frac{\lambda(t)}{\lambda(s)}\right)+\left(\frac{\lambda(s)}{\lambda(t)}\right)^{2}+\frac{\lambda(s)}{\lambda(t)} \bar{K}^{2}$

$\bar{K}=K(t) \lambda(t) / \lambda(s)$ for $0 \leqslant s \leqslant t_{1}$, while $\bar{K}$ does not simplify for $t_{1} \leqslant s \leqslant t$.

The integral in equation (8) reduces to the sum of integrals over the three intervals in equation (10) on which the stretch and shear history is defined. It will be convenient to define a special notation for the contribution from the integral on the interval $-\infty<s<0$. Introducing a change of variables in this integral, and noting by equations $(9)$ and $(11)$ that the arguments $\hat{I}_{x}(t)$ in the integrand depend on $\lambda(t)$ and $K(t)$, we first write:

$$
G_{x}(\lambda(t), K(t), t)=\int_{i}^{\infty} U_{x}\left(\hat{I}_{1}(t), \hat{I}_{2}(t), s\right) \mathrm{d} s
$$

and then let:

$$
G(\lambda(t), K(t), t)=G_{1}(\lambda(t), K(t), t)+\lambda^{-1}(t) G_{2}(\lambda(t), K(t), t)
$$

The relevant stress components at each time $t>t_{1}$ are given by the expressions:

$$
\sigma_{13}(t)=K(t) \lambda^{2}(t) G(\lambda(t), K(t), t)+K(t) \lambda(t) \int_{0}^{t_{1}}\left[U_{1}+\frac{\lambda(s)}{\lambda(t)} U_{2}\right] \frac{\lambda(t)}{\lambda^{2}(s)} \mathrm{d} s
$$$$
+\int_{i_{1}}^{t}\left[U_{1}+\frac{\lambda(s)}{\lambda(t)} U_{2}\right]\left[K(t) \frac{\lambda(t)}{\lambda(s)}-K(s) \sqrt{\frac{\lambda(s)}{\lambda(t)}}\right] \frac{\lambda(t)}{\lambda(s)} \mathrm{d} s
$$$$
\sigma_{11}(t)-\sigma_{22}(t)=\left[\lambda^{2}(t)-\frac{1}{\lambda(t)}\right] G(\lambda(t), K(t), t)
$$$$
+\int_{0}^{t}\left[U_{1}+\frac{\lambda(s)}{\lambda(t)} U_{2}\right]\left[\left(\frac{\lambda(t)}{\lambda(s)}\right)^{2}-\frac{\lambda(s)}{\lambda(t)}\right] \mathrm{d} s
$$$$
-K^{2}(t) \lambda(t) G_{2}(\lambda(t), K(t), t)-K^{2}(t) \lambda(t) \int_{0}^{t_{1}} U_{2} \frac{1}{\lambda(s)} \mathrm{d} s
$$$$
-\int_{t}^{t} U_{2} \frac{\lambda(s)}{\lambda(t)}\left[K(t) \frac{\lambda(t)}{\lambda(s)}-K(s) \sqrt{\frac{\lambda(s)}{\lambda(t)}}\right]^{2} \mathrm{~d} s
$$

Since only small amplitude torsional oscillations are considered, $|\phi(s)| \ll 1$. This implies, from equations (2) and (5) that $|\bar{K}| \ll 1$. Approximate expressions for the stresses are obtained by neglecting terms of order $|\bar{K}|^{2}$ in equations (11)-(15). Furthermore, equations (2) and (5) also imply that $\sigma_{13}(t)$ and $\sigma_{11}(t)-\sigma_{22}(t)$ depend on $r$ through their dependence on $K$. If terms of $\mathrm{O}\left(\bar{K}^{2}\right)$ are neglected, it follows from equation (7) that the invariants become independent of $r$ and from equation (9), so does $U_{x}$. The expression for $\sigma_{13}(t)$ is given in equation (14) where the first term is now $K(t) \lambda^{2}(t) G(\lambda(t), 0, t)$. The expression for $\sigma_{11}(t)-\sigma_{22}(t)$ reduces to:

$$
\begin{aligned}
\sigma_{11}(t) & -\sigma_{22}(t)=\left[\lambda^{2}(t)-\lambda^{-1}(t)\right] G(\lambda(t), 0, t)+\int_{0}^{t}\left[U_{1}+\frac{\lambda(s)}{\lambda(t)} U_{2}\right] \\
\times & {\left[\left(\frac{\lambda(t)}{\lambda(s)}\right)^{2}-\left(\frac{\lambda(s)}{\lambda(t)}\right)\right] \mathrm{d} s }
\end{aligned}
$$

and is independent of $r$.
To this point, $\lambda(s)$ is an arbitrary axial stretch history. We now consider a step stretch history, i.e. $\lambda(s)=\lambda(t)$ for $0 \leqslant s \leqslant t$. The invariant arguments of the integrals over this range become, from equation (15), $I_{1}(t, s)=I_{2}(t, s)=3$. Equations (13) and (16) simplify to:

$$
\begin{aligned}
\sigma_{13}(t)= & K(t) \lambda(t)\left\{\frac{\lambda(t)\left[\sigma_{11}(t)-\sigma_{22}(t)\right]}{\lambda^{2}(t)-\lambda^{-1}(t)}+\frac{1}{\lambda(t)} \int_{0}^{t_{1}}\left[U_{1}\right.\right. \\
& \left.\left.+U_{2}\right] \mathrm{~d} s\right\}+\int_{t^{t}}^{t}\left[U_{1}+U_{2}\right][K(t)-K(s)] \mathrm{d} s
\end{aligned}
$$

where

$$
U_{\alpha}=U_{\alpha}(3,3, t-s), \quad \alpha=1,2
$$

Let $G(t),(G(\infty)=0)$, denote the shear stress relaxation function of the linear viscoelastic constitutive equation. We also introduce the notation $G_{*}(s)=\mathrm{d} G(s) / \mathrm{d} s$. It can be shown that:

$$
U_{1}(3,3, s)+U_{2}(3,3, s)=-G_{*}(s)
$$

and from equations (11) and (13) that:

$$
G(1,0, t)=G(t)
$$

Equation (17) can now be rewritten as:

$$
\begin{aligned}
\sigma_{13}(t)= & K(t) \lambda(t)\left\{\frac{\lambda^{2}(t)\left[\sigma_{11}(t)-\sigma_{22}(t)\right]}{\lambda^{3}(t)-1}-\frac{G(t)-G(0)}{\lambda(t)}\right. \\
& \left.+\frac{1}{\lambda(t)} \int_{t^{\prime}}^{t} G_{*}(t-s) \frac{K(s)}{K(t)} \mathrm{d} s\right\}
\end{aligned}
$$

We now consider the calculation of quantities associated with the deformed cylinder. Since $\sigma_{11}(t)-\sigma_{22}(t)$ is independent of $r$, the scalar $p(t)$ can be chosen so that $\sigma_{22}(t)=0$ throughout the cylinder. The axial force $N(t)$ on the ends of the cylinder is given by:

$$
N(t)=\sigma_{11}(t) A(t)=\frac{\sigma_{11}(t) A_{0}}{\lambda(t)}
$$

where $A_{0}$ is the original cross-sectional area. The twisting moment $T(t)$ is given by:

$$
T(t)=\int_{a(t)}^{b(t)} 2 \pi \sigma_{13} r^{2} \mathrm{~d} r
$$

where $a(t)$ and $b(t)$ are the inner and outer radii of the deformed cylinder at time $t$.

Let equation (23) be evaluated using equation (21) where $K(s)=K(r, s)$ is given in equation (2). When it is simplified using equation (22) and then expressed in terms of the original dimensions using $a(t)=a_{0} / \sqrt{\lambda(t)}$, etc., the

$$
\frac{T(t)}{\phi(t)}=\frac{J_{0}}{L_{0}}\left\{\frac{\lambda N(t)}{A_{0}\left(\lambda^{3}-1\right)}-\frac{G(t)-G(0)}{\lambda^{3}}+1 \lambda^{3} \int_{i}^{t} G_{*}(t-s) \frac{\phi(s)}{\phi(t)} \mathrm{d} s\right\}
$$


where $J_{0}=\pi\left(b_{0}^{4}-a_{0}^{4}\right) / 2$ is the polar moment of inertia associated with the undeformed state.

Equation (24) represents the generalization for the BKZ constitutive equation of Rivlin's formula for nonlinear elasticity. In particular, if a step extension and a step twist are applied simultaneously, i.e. $t_{1}=0$ and $\phi(s)=\phi(t)$, then the terms involving $G(t)$ drop out. The remaining expression, which agrees with that of Rivlin ${ }^{4}$, does not depend on any material parameters. If the step twist is applied at time $t_{1}$ after the step extension, equation (24) shows that the correction to this result is additive and depends on the shear relaxation function of linear viscoelasticity and the axial stretch.

\section{ANALYSIS OF THE EXPERIMENT}

The experiment configuration in ref. 1 consisted of a flywheel which rotated about a vertical axis, and was attached to viscoelastic hollow cylinders at its upper and lower surfaces. The other ends of these cylinders were fixed. Natural rubber and plasticized PVC specimens were used. In terms of the twisting moment $T$ and angular displacement $\phi$, the equation of angular motion of the flywheel is:

$$
I \frac{d^{2} \phi}{\mathrm{d} t^{2}}=-2 T
$$

where $I$ is the moment of inertia of the flywheel. Using equation (24), the following integral-differential equation is obtained for $\phi(t)$ :

$$
\begin{array}{r}
\frac{d^{2} \phi}{\mathrm{d} t^{2}}+\frac{2 J_{0}}{I L_{0}}\left\{\frac{\lambda N}{A_{0}\left(\lambda^{3}-1\right)} \phi+\frac{G(0)-G(t)}{\lambda^{3}} \phi+\frac{1}{\lambda^{3}}\right. \\
\left.\int_{t_{1}}^{t} G_{*}(t-s) \phi(s) \mathrm{d} s\right\}=0
\end{array}
$$

In the experiment, the viscoelastic cylinders were given a step axial stretch, followed by a step angular displacement $\phi_{0}$ after time interval $t_{1}$ and then released. Thus, $\phi\left(t_{1}^{+}\right)=\phi_{0}, \phi\left(t_{1}^{+}\right)=0$. The period of the subsequent oscillatory motion was measured by averaging over 20 oscillations. This implies that the damping can be assumed to be relatively small. Furthermore, the axial force relaxation $N(t)$ was slow relative to the period of oscillation.

The transient solution to equation (26) which satisifies the initial conditions has been discussed to a limited extent by Markowitz ${ }^{8}$. This discussion and the experimental results suggest that after a short time the solution can be approximated by $\phi(t)=\phi_{0} e^{i / t)}$, where $\omega$ is a constant, possibly complex-valued.

Substitution of this expression into equation (26) leads to the following combination of terms:

$$
\phi_{0} e^{\mathrm{j}(\nu) t}\left[G(0)+\int_{0}^{t-t_{1}} G_{*}(\alpha) e^{-\mathrm{j}(r) x} \mathrm{~d} \alpha\right]
$$

Let $\bar{G}\left(\omega, t-t_{1}\right)$ denote the expression in brackets in equation (27). As $t-t_{1} \rightarrow \infty, \bar{G}\left(\omega, t-t_{1}\right)$ approaches the complex shear modulus of linear viscoelasticity, $G(\omega)=G^{\prime}(\omega)+i G^{\prime \prime}(\omega)$. Experimenters take the value of $G(\omega)$ to be that which is measured after a finite number of cycles. This corresponds to the value of $\bar{G}\left(\omega, t-t_{1}\right)$ after same time interval $t-t_{1}$. The number of cycles or the time interval is chosen to be sufficiently large that the transients induced by the initiation of oscillations are very small. Since the period in the experiment has been measured over_several oscillations, we now take as $G(\omega)$ the value of $\bar{G}\left(\omega, t-t_{1}\right)$ for some appropriate time intervale $t-t_{1}$. The equation for the constant $\omega$ becomes:

$$
-\omega^{2}+\frac{2 J_{0}}{I L_{0}}\left\{\frac{\lambda N}{A_{0}\left(\lambda^{3}-1\right)}-\frac{G(t)}{\lambda^{3}}+\frac{1}{\lambda^{3}}\left[G^{\prime}(\omega)+i G^{\prime \prime}(\omega)\right]=0\right.
$$

Since a large number of oscillations are observed, the damping must be small. This suggests that $\left|G^{\prime \prime}(\omega)\right| /\left|G^{\prime}(\omega)\right| \ll 1$ and that the roots of equation (28) lie close to the real axis. Since only the period, or real part of $\omega$ is of interest here, equation (28) can be further reduced. In terms of the period of oscillation $p=2 \pi / \omega$, equation (28) becomes:

$$
\frac{C}{p^{2}}=\frac{\lambda N}{A_{0}\left(\lambda^{3}-1\right)}-\frac{G(t)}{\lambda^{3}}+\frac{G^{\prime}(\omega)}{\lambda^{3}}
$$

where

$$
C=(2 \pi)^{2} I L_{0} / 2 J_{0}
$$

The work reported in ref. 1 tests the hypothesis that $1 / p^{2}$ is proportional to the first term in equation (29), which would be the case if the material were modelled as nonlinearly elastic or 'time dependent elastic'. Although the experimental results in Figure 2, (for natural rubber), of ref. 1 suggest that $1 / p^{2}$ and $\lambda N\left(\lambda^{3}-1\right)^{-1}$ lie on a straight line, this line does not pass through the origin. There is a

* There does not yet appear to be a study of the convergence of $\bar{G}(\omega, t)$ to $G(\omega)$ as $t \rightarrow \infty$ in linear viscoelasticity. From a different viewpoint, there is no measure as to how close $\bar{G}(\omega, 2 \pi n / \omega)$, the value after $n$ cycles, is to $G(\omega)=\bar{G}(\omega, \infty)$

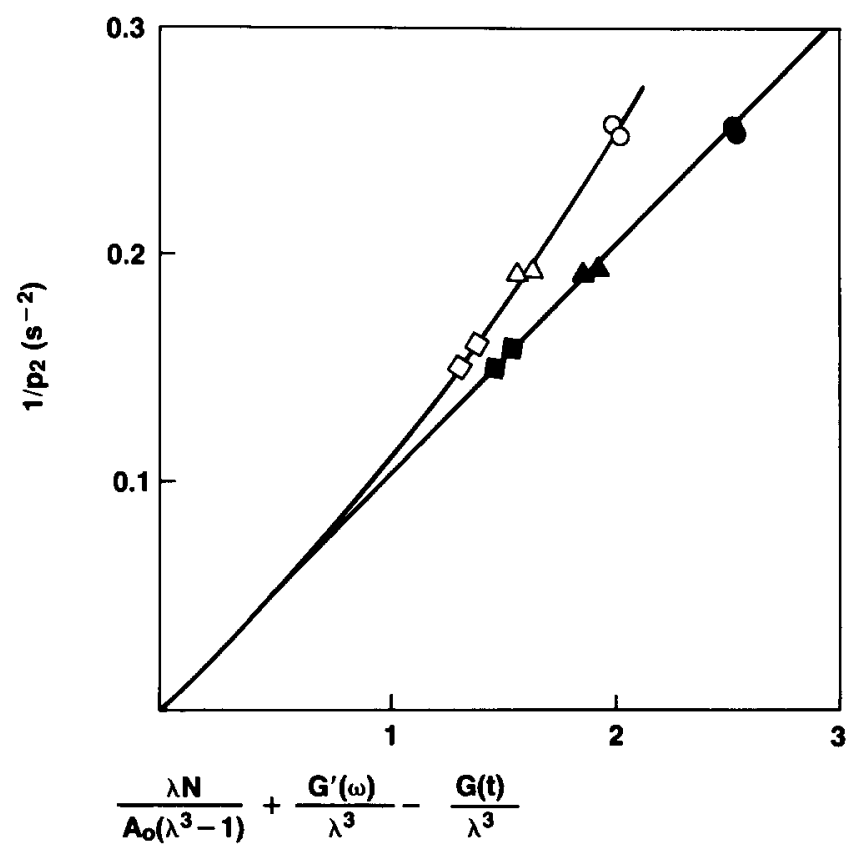

Figure 2 Comparison of experimental results for natural rubber using relation (29) (solid symbols) and using only the first term (open symbols).

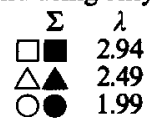


discrepancy of about $10 \%$ which is attributed ${ }^{1}$ to the rate dependence of the natural rubber. The present work takes this into consideration by rederiving the relation for a specific model of a simple material with memory, the BKZ fluid. This leads to the additional terms in equation (29). It is now shown that equation (29) can account for the discrepancy.

Since the experiment was viewed in the context of elasticity, data was not taken which could be used to analyse it in terms of viscoelasticity. We can however make reasonable assumptions about the viscoelastic properties of the rubber used.

The first term in equation (29) represents the contribution of axial stress relaxation due to a step axial stretch. From equation (22), this can be written as:

$$
\frac{\lambda N}{A_{0}\left(\lambda^{3}-1\right)}=\frac{\sigma_{11}-\sigma_{22}}{\lambda\left(\lambda^{2}-\frac{1}{\lambda}\right)}
$$

where $\sigma_{22}=0$. Laboratory stress relaxation data on vulcanized butyl rubber and on cross linked natural rubber shows that the reduced stress $\left(\sigma_{11}-\sigma_{22}\right) /\left(\lambda^{2}-\lambda^{-1}\right)$ has, to a very good approximation, the same time dependence for different values of $\lambda$. This suggests that the reduced stress can be written in the product form $f(\lambda) G(t)$. Thus, from equation (16) with $\lambda(s)=\lambda(t)$ and equation $(20)$ :

$$
\left.\frac{\sigma_{11}-\sigma_{22}}{\lambda^{2}-\frac{1}{\lambda}}=G(\lambda, 0, t)=f(\lambda) G(t), f(1)=1\right)
$$

Equation (29) can now be written:

$$
\frac{C}{p^{2}}=\frac{f(\lambda) G(t)}{\lambda}\left[1-\frac{1}{\lambda^{2} f(\lambda)}\right]+\frac{G^{\prime}(\omega)}{\lambda^{3}}
$$

It is assumed that equation (31) also holds for the rubber used in ref. 1. From our data on natural rubber and from data by Rivlin and Saunders ${ }^{5}$, we can obtain values for $\left[1-1 / \lambda^{2} f(\lambda)\right]$.

Data for the analysis of equation (32) was obtained from Figure 2 of ref. 1. This Figure shows that four oscillation tests were carried out at each fixed value of $\lambda$. From this Figure it was also possible to calculate the force relaxation and therefore $f(\lambda)$ and $G(t)$.

We estimated that the first data point was taken $100 \mathrm{~s}$ after the axial step stretch was applied. A reasonable estimate for the duration of these four sets of data then implied that $G(t)$ had the same order of magnitude of stress relaxation as butyl rubber, (about $12 \%$ per decade). As a result, the relaxation property of butyl rubber was taken as a model for that of the rubber used in ref. 1 . Finally, since for slowly relaxing materials $G^{\prime}(\omega) \approx G(t)$, where $t=1 / \omega$, the value of $G^{\prime}(\omega)$ could be estimated. Since the variation of $\omega$ was small over the data given, $G^{\prime}(\omega)$ was assumed to be the same for all runs. Accordingly, $G^{\prime}(\omega)=8$.

Results are shown in Figure 2. The solid symbols represent a plot of $1 / p^{2}$ versus the right hand side of equation (32). The open symbols represent a plot of $1 / p^{2}$ versus $\lambda N\left(\lambda^{3}-1\right)^{-1}$ as taken from ref. 1 . Note that the solid symbols lie on a straight line which passes through the origin, whereas this is not possible for the open symbols. Thus, the additional terms introduced by the $\mathrm{BKZ}$ model provide the needed correction.

\section{FORCED VIBRATIONS}

In this section we study the response of a cylinder which has been subjected to forced torsional oscillations following an axial step stretch history. The relation between the shear stress and a general shear strain history, based on the current length of the specimen, is given by equation (21). On substituting $K(t)=K_{0} e^{i(s)}$, where $\omega$ is a real constant, into equation (21), we obtain the following relation which is appropriate to the current conditions:

where

$$
\sigma_{13}(t)=G(\lambda, t ; \omega) K(t)
$$

$$
G(\lambda, t ; \omega)=G^{\prime}(\lambda, t ; \omega)+i G^{\prime \prime}(\lambda, t ; \omega)
$$

is a modified complex shear modulus. The dependence on $\lambda$ arises because of the axial step stretch and on $t$ because of the corresponding axial stress relaxation. It is defined in terms of these quantities by the relations:

$$
\begin{gathered}
G^{\prime}(\lambda, t ; \omega)=\frac{\lambda^{2}\left(\sigma_{11}-\sigma_{22}\right)}{\lambda^{2}-1 / \lambda}-G(t)+G^{\prime}(\omega) \\
G^{\prime \prime}(\lambda, t, \omega)=G^{\prime \prime}(\omega)
\end{gathered}
$$

where $G^{\prime}(\omega)+i G^{\prime \prime}(\omega)$ is the complex shear modulus of linear viscoelasticity.

Note that according to equation (35), the imaginary part of the complex modulus is independent of the stretch of the cylinder.

\section{REFERENCES}

1 Rosen, R. H., Kolsky, H. and Pipkin, A. C. J. Appl. Phys. 1975, 46, 4441

2 Min, B. K., Kolsky, H. and Pipkin, A. C. Int. J. Solid Structures 1977, 13, 771

3 Crissman, J. M. and Zapas, L. J. J. Appl. Phys. 1977, 48, 4049

4 Rivlin, R. S. Phil. Trans. R. Soc. Lond. 1949, A243, 173

5 Rivlin, R. S. and Saunders, D. W. Phil. Trans. R. Soc. Lond. 1951, A243, 251

6 Bernstein, B., Kearsley, E. A. and Zapas, L. J. Trans. Soc. Rheol. 1963 7, 391

7 Bernstein, B. Acta Mechanica 1966, 11, 329

8 Markowitz, H. J. Appl. Phys. 1963, 34, 21 